\title{
Ultra-wide angle lens design with relative illumination analysis
}

W.-S. Sun

C.-L. Tien

cltien@fcu.edu.tw

Y.-H. Chen

P.-Y. Chu
Department of Optics and Photonics, National Central University, Chung-Li 32001, Taiwan

Department of Electrical Engineering, Feng Chia University, Taichung 40724, Taiwan

Department of Optics and Photonics, National Central University, Chung-Li 32001, Taiwan

Department of Optics and Photonics, National Central University, Chung-Li 32001, Taiwan

An ultra-wide angle lens design with relative illumination analysis is presented. The half field angle of $80^{\circ}$, the relative illumination of the image plane will be reduced. It is necessary to increase the image numerical aperture ratio for $X$ and $Y$ direction, and decrease the angle between the chief ray and optical axis in the image space. However the ultra-wide angle lens induces great barrel distortion causing image extrusion. Therefore, we use F-theta distortion to replace optical distortion. Seven lenses and two pieces of flat glass are used for a sensor $1 / 6^{\prime \prime}$ sensor with 2.1 million pixels. The final result for the lens is a half viewing angle of $80^{\circ}, \mathrm{F} / 2.4$, a focal length $1.1 \mathrm{~mm}$, a length of $22.37 \mathrm{~mm}$, F-theta distortion of less than $2 \%$, and relative illumination greater than $83 \%$.

[DOI: http://dx.doi.org/10.2971/jeos.2016.16001]

Keywords: Lens design, relative illumination, numerical aperture, F-theta distortion, ultra-wide angle lens

\section{INTRODUCTION}

Generally, optical lenses with half field angle greater than $60^{\circ}$ are called ultra-wide angle lenses. In the fisheye lenses half field angle is equal to or exceeds $90^{\circ}$ called. In 1919 R. W. Wood [1] used a pinhole in front of a container filled with water as the prototype of the first ultra-wide angle lens, simulating the fisheye image in the water. Many improvements have been made to the ultra-wide angle lens design since that time. For example, in 1922 Bond [2] replaced Wood's container filled with water with hemispherical glass lenses to achieve the same effect, but this produced large aberration. Beck [3] and Hill [4] added one or two lenses to improve on the Bonds aberration. Through continuous improvement, the current generation of ultra-wide angle lenses has been used in many places, such as: J. Y. Zheng [5] using a fisheye lens to capture the image and perform street panorama image processing. Furthermore, ultra-wide angle lenses are also using in unmanned remotely controlled vehicles [6] and in car recorder lens. A fisheye lens with broad spectrum is used for detecting the rice canopy or other plant growth scenarios [7]. W. Li [8] presented a panoramic stereo imaging system which uses a single camera coaxially combined with a fisheye lens and a convex mirror.

Ultra-wide-angle lenses have been discussed in a large number of related studies papers and give rise to numerous patents [9]-[14]. The requirements are as follows; half field angle ranging from $70^{\circ}$ to $90^{\circ}$, and a number of elements ranging from 7 to 11; the overall length of the lens is from $25 \mathrm{~mm}$ to $40 \mathrm{~mm}$, and the f number is 2.8. Design specifications used in this study include an $80^{\circ}$ half field angle, 7 lenses with a length $22.37 \mathrm{~mm}$, and $\mathrm{F} / 2.4$.

\section{METHOD}

The relative illumination and F-theta distortion must be considered in the ultra-wide angle lens design.

\subsection{Relative illumination}

The relative illumination is related to the NA of the image space and the $\cos ^{4}$ law. In order to calculate the NA value, five reference rays should be defined.

\subsubsection{Definition of reference rays}

Figure 1 illustrates the definition of the five reference rays (R1, R2, R3, R4, and R5) [15]. We set the light source at the $-Y$ height of the object point on the object plane. The Z-axis is the optical axis. The ray passing through the center of the entrance pupil is defined as the chief ray R1; $\theta$ is the half field angle between the chief ray $\mathrm{R} 1$ and the optical axis in object space. We denote the four marginal rays R2, R3, R4, R5, which pass through the $+Y,-Y,+X$ and $-X$ of the entrance pupil. 


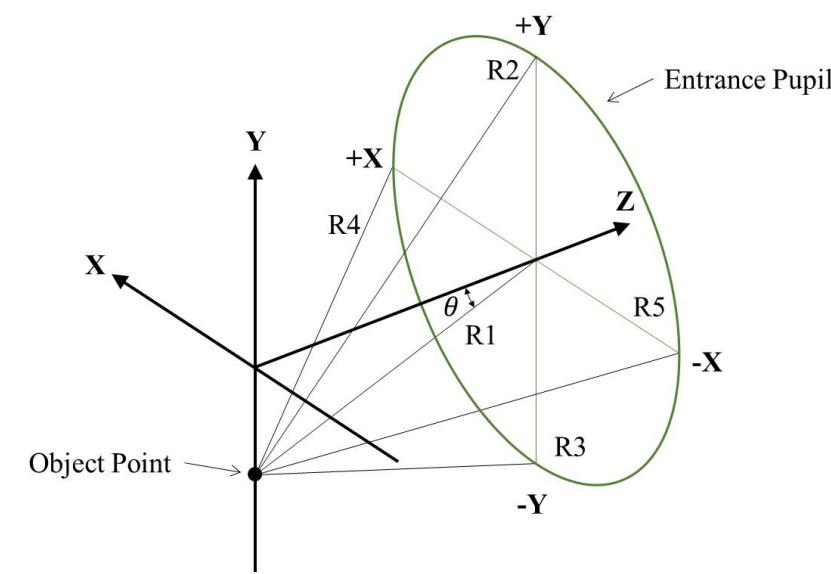

FIC. 1 The specification of the sensor

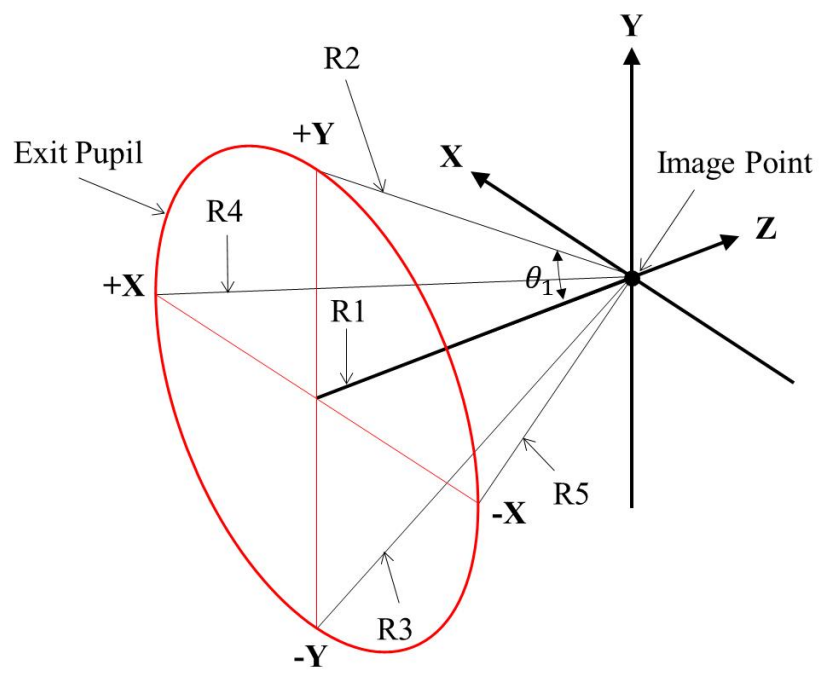

FIG. 2 The axial numerical aperture.

\subsubsection{Calculation of the image numerical aperture}

When the half field angle $\theta$ is zero, the chief ray R1 is located on the optical axis. The five reference rays from the exit pupil to the image plane are shown in Figure 2 and $\theta_{1}$ is the aperture angle between R2 and R1 in the image space. The aperture angles between the chief ray $\mathrm{R} 1$ and marginal rays (R2, R3, $\mathrm{R} 4$, and R5) are the same at the zero field angle. Therefore, the axial numerical aperture in the image space (NA0) is defined as

$$
N A_{0}=n \sin \theta_{1}
$$

where $n$ is the refractive index of the image space.

When the half field angle $\theta$ is not zero, the numerical aperture is different in the $\mathrm{X}$ and $\mathrm{Y}$ direction. The off-axis $\mathrm{Y}$ direction reference rays are shown in Figure 3. Here is the chief angle between R1 and optical axis in the image space; $\theta_{2}$ is the aperture angle between R2 and R1 in the image space; and $\theta_{3}$ is the aperture angle between R3 and R1 in the image space.

Since aberrations have different influences on the angles of $\theta_{2}$ and $\theta_{3}$. We can calculate the average angle of $\theta_{2}$ and $\theta_{3}$. Thus the numerical aperture of $Y$ direction $\left(N A_{Y}\right)$ is given by

$$
N A_{Y}=n \sin \frac{\theta_{2}+\theta_{3}}{2} .
$$

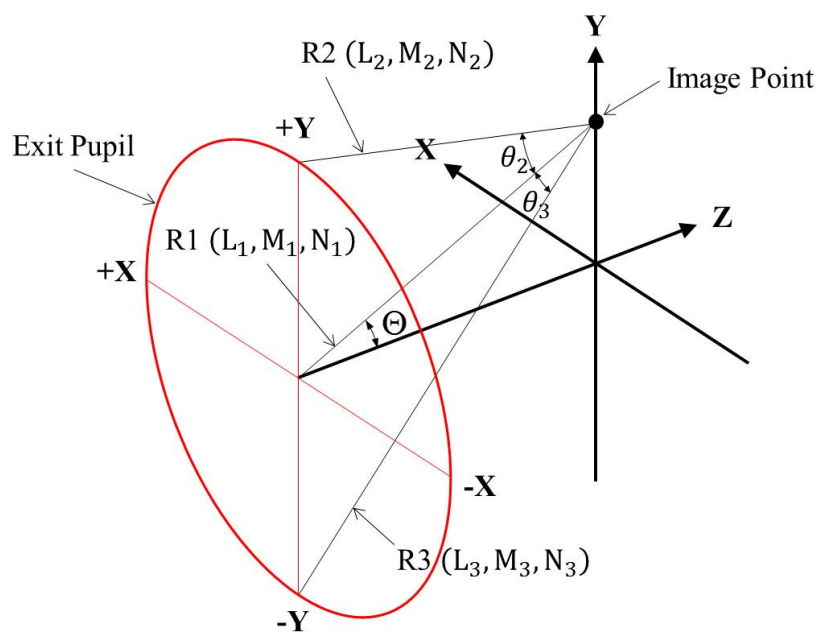

FIG. 3 The off-axis $Y$ direction reference rays.

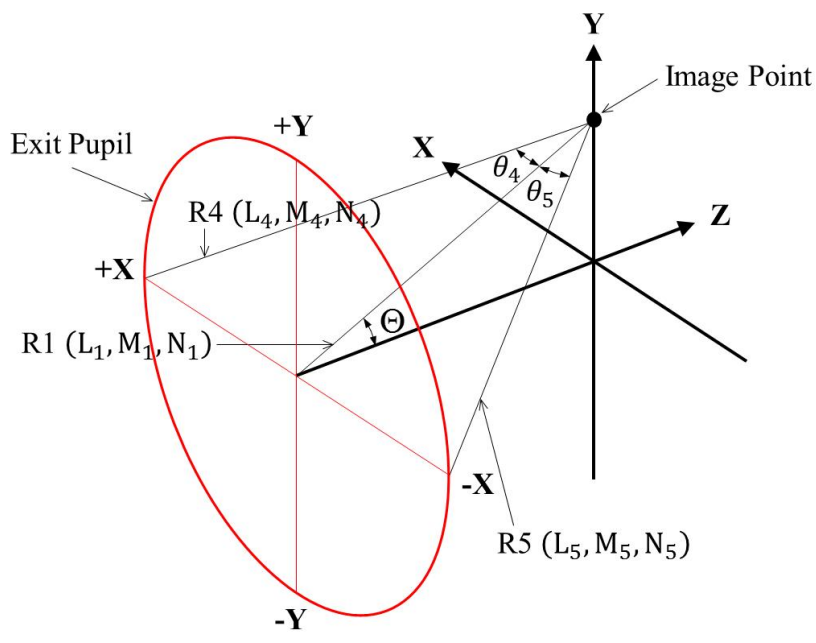

FIG. 4 The off-axis $X$ direction reference rays.

The directional cosines $(\mathrm{L}, \mathrm{M}, \mathrm{N})$ of $\mathrm{R} 1, \mathrm{R} 2$ and $\mathrm{R} 3$ are different. They are R1 (L1, M1, N1), R2 (L2 M2, N2) and R3 (L3, M3, N3) respectively. The directional cosine is the unit victor, and $\theta_{2}$ and $\theta_{3}$ are calculated by Eq. (3) and (2.1.2).

$$
\begin{array}{r}
\theta_{2}=\cos ^{-1}\left(\frac{\mathrm{L}_{1} \mathrm{~L}_{2}+\mathrm{M}_{1} \mathrm{M}_{2}+\mathrm{N}_{1} \mathrm{~N}_{2}}{\sqrt{\mathrm{L}_{1}^{2}+\mathrm{M}_{1}^{2}+\mathrm{N}_{1}^{2}} \sqrt{\mathrm{L}_{2}^{2}+\mathrm{M}_{2}^{2}+\mathrm{N}_{2}^{2}}}\right) \\
=\cos ^{-1}\left(\mathrm{~L}_{1} \mathrm{~L}_{2}+\mathrm{M}_{1} \mathrm{M}_{2}+\mathrm{N}_{1} \mathrm{~N}_{2}\right) \\
\theta_{3}=\cos ^{-1}\left(\mathrm{~L}_{1} \mathrm{~L}_{3}+\mathrm{M}_{1} \mathrm{M}_{3}+\mathrm{N}_{1} \mathrm{~N}_{3}\right)
\end{array}
$$

The off-axis $X$ direction reference rays are shown in Figure 4. Here $\theta_{4}$ is the aperture angle between R4 and R1; $\theta_{5}$ is the aperture angle between R5 and R1.

Because the system is axial symmetric, the angles of $\theta_{4}$ and $\theta_{5}$ have the same magnitude. Therefore, the numerical aperture for the $\mathrm{X}$ direction $\left(N A_{\theta} \mathrm{X}\right)$ is defined as

$$
N A_{\mathrm{X}}=n \sin \theta_{4}
$$

The direction cosine of $\mathrm{R} 4$ is (L4, M4, N4), and $\theta_{4}$ is given by

$$
\theta_{4}=\cos ^{-1}\left(\mathrm{~L}_{1} \mathrm{~L}_{4}+\mathrm{M}_{1} \mathrm{M}_{4}+\mathrm{N}_{1} \mathrm{~N}_{4}\right)
$$




\subsubsection{Method for estimating the relative illumination}

The illumination of the object in the image space, as proposed by Burle [16], is found as shown in Eq. (7)

$$
E_{\theta}=E_{\mathrm{obj}} R \mathrm{~T}_{\mathrm{L}} N A_{\theta} 2 \cos ^{4} \Theta,
$$

where $E_{\theta}$ is the illumination of the image point from the incidence ray of the half field angle $\theta$ in the object space; $E_{\text {obj }}$ is illumination of object; $R$ is the reflectivity of the object; $T_{L}$ is the penetration of the lens; $\Theta$ is the chief angle in the image space; $N A_{\theta}$ is the numerical aperture of the image point from the incidence ray of the half field angle $\theta$.

The relative illumination $(R I)$ can be written as

$$
R I=\frac{E_{\theta}}{E_{0}}=\frac{\left(N A_{\theta}\right)^{2} \times \cos ^{4} \Theta}{\left(N A_{0}\right)^{2}},
$$

where $E_{0}$ is the illumination of the center image point from the incidence ray for a half field angle of $0^{\circ}$.

When $\theta$ becomes large, there will be large differences in its $N A_{\mathrm{X}}$ and $N A_{\mathrm{Y}}$ in the image space [17]. The relative illumination can be modified as in Eq. (9).

$$
R I=\frac{E_{\theta}}{E_{0}}=\frac{N A_{Y} N A_{\mathrm{X}} \times \cos ^{4} \Theta}{\left(N A_{0}\right)^{2}}
$$

Finally, we can simplify this to obtain Eq. (10).

$$
R I(\theta)=\frac{\sin \left(\frac{\theta_{2}+\theta_{3}}{2}\right) \sin \theta_{4} \times \cos ^{4} \Theta}{\sin \theta_{1}^{2}}
$$

It should be noted that the optimization process is performed to increase the angles of $\theta_{2}, \theta_{3}$, and $\theta_{4}$, respectively, and to reduce the angle of $\Theta$.

\subsection{F-theta distortion and calculation of the depth of field}

The optical distortion is large and cannot be eliminated by the lens design with a large half field angle. It will cause the edge images close together and cannot be distinguished, so we use the F-theta distortion design to expand the edge images with a good recognition.

The F- $\theta$ distortion [18] is given by

$$
F-\theta \text { distortion }=\frac{h^{\prime}-h}{h} \times 100 \%,
$$

where $h^{\prime}$ is the real image height; and $h$ is the ideal image height as

$$
h=f \times \theta,
$$

where $f$ is the focal length of the lens; and $\theta$ is the half field angle for which the unit is radians.

\section{DESIGN RESULTS}

\subsection{Specifications of sensor and lens}

We use the OV2722 sensor with 2.1 million pixels. The pixel size of the sensor is $1.4 \mu \mathrm{m}$ and the specification is shown in Table 1. The requirement of an ultra-wide angle lens design is shown in Table 2. In object space, a half field angle of $80^{\circ}$, $\mathrm{F} / 2.4$, and a focal length $1.1 \mathrm{~mm}$ are specified in our design.

\begin{tabular}{|l|l|}
\hline Parameters & Specification \\
\hline Model & OV2722 \\
\hline Resolution & $1932 \times 1092$ \\
\hline Active Area & $2.73 \mathrm{~mm} \times 1.53 \mathrm{~mm}$ \\
\hline Device Diagonal & $3.13 \mathrm{~mm}$ \\
\hline Pixel Pitch & $1.4 \mu \mathrm{m}$ \\
\hline
\end{tabular}

TABLE 1 The specification of the sensor.

\begin{tabular}{|l|c|}
\hline Items & Design Requirements \\
\hline Number of lens (without plate) & 7 \\
\hline F/\# & 2.4 \\
\hline Total length & $<25 \mathrm{~mm}$ \\
\hline Half field angle & $80^{\circ}$ \\
\hline Effective focal length & $1.1 \mathrm{~mm}$ \\
\hline MTF(178 lp/mm) & $>40 \%$ \\
\hline Relative illumination & $>80 \%$ \\
\hline Lateral color & $<1.4 \mu \mathrm{m} / \mathrm{pixel}$ \\
\hline F-theta distortion & $<5 \%$ \\
\hline
\end{tabular}

TABLE 2 The requirement of the ultra-wide angle lens design.

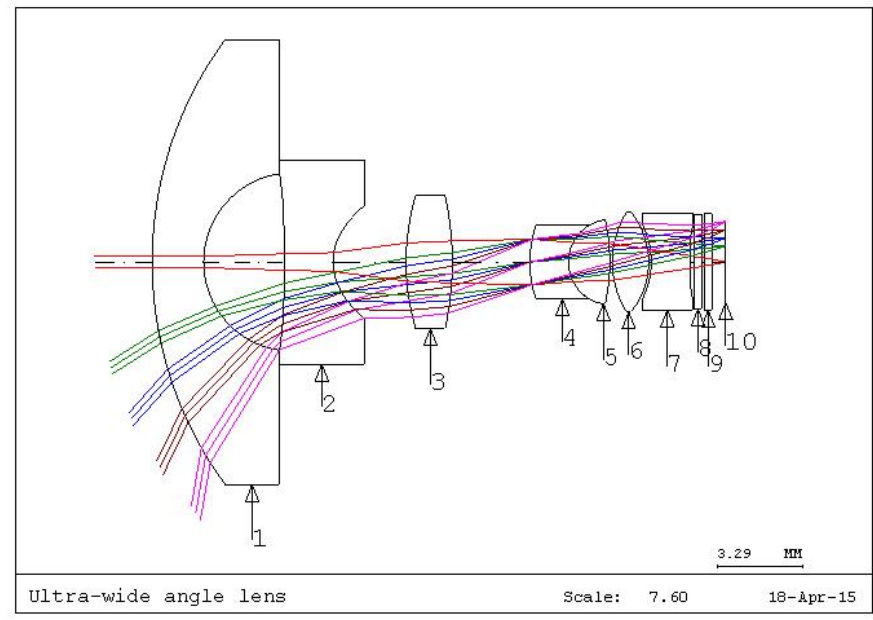

FIG. 5 Lens layout of ultra-wide angle lens design.

\subsection{Lens layout and image quality}

The ultra-wide angle lens is shown in Figure 5. There are seven lenses and two pieces of plate glass. Wherein the first, third, fourth, the fifth, sixth glasses are spherical lens, the second and the seventh components are plastic aspheric lenses, the eighth piece of flat glass is the IR-Cut filter, and the ninth piece of flat glass is a cover glass for the sensor. Table 3 shows design data for ultra-wide angle lens. Table 4 lists its aspheric coefficients.

The pixel size of the sensor is $1.4 \mu \mathrm{m}$. We chose 4 pixels width as one line pair (lp), so the space frequency is $178 \mathrm{lp} / \mathrm{mm}$. Figure 6 shows the MTF plot of the spatial frequency $178 \mathrm{lp} / \mathrm{mm}$ for different field of view, the minimum value of its MTF curve at $178 \mathrm{lp} / \mathrm{mm}$ position is 0.429 for 1.0 field tangent direction. Figure 7 shows the F- $\theta$ distortion. The ordinate is half field angle from $0^{\circ}$ to $80^{\circ}$ in object side, the abscissa is F- $\theta$ distortion $(\%)$, and the maximum of F- $\theta$ distortion is $2.02 \%$ located at the half field angle of $80^{\circ}$.

Lateral chromatic aberration is the imaging height difference 


\begin{tabular}{|c|c|c|c|c|}
\hline No & Type & Radius & Thickness & Glass \\
\hline Object & Sphere & infinity & infinity & \\
\hline 1 & Sphere & 14.3765 & 2.00867 & 6968.5541 \\
\hline 2 & Sphere & 3.44135 & 3.14572 & \\
\hline 3 & Asphere & -59.463 & 1.89576 & 5252.60000 \\
\hline 4 & Asphere & 2.05825 & 2.85213 & \\
\hline 5 & Sphere & 9.12841 & 1.8 & 7174.2962 \\
\hline 6 & Sphere & -11.893 & 3.02629 & \\
\hline Stop & Sphere & 4.32817 & 1.5 & 7847.2608 \\
\hline 8 & Sphere & 1.64497 & 1.61405 & 6127.5863 \\
\hline 9 & Sphere & -7.5167 & 0.1 & \\
\hline 10 & Sphere & 3.58107 & 1.4197 & 5399.5971 \\
\hline 11 & Sphere & -3.0231 & 0.1 & \\
\hline 12 & Asphere & -2.9929 & 1.5 & 5674.3481 \\
\hline 13 & Asphere & 15.4372 & 0.17212 & \\
\hline 14 & Sphere & infinity & 0.3 & 5168.6417 \\
\hline 15 & Sphere & infinity & 0.1 & \\
\hline 16 & Sphere & infinity & 0.3 & 5168.6417 \\
\hline 17 & Sphere & infinity & 0.53175 & \\
\hline Image & Sphere & infinity & 0 & \\
\hline \multicolumn{5}{r}{} \\
\hline
\end{tabular}

TABLE 3 The lens data of the ultra-wide angle lens design.

\begin{tabular}{|l|l|l|l|}
\hline Surface & Conic constant & 4th order & 6th order \\
\hline 3 & -100 & -0.00072705 & 0 \\
\hline 4 & -0.995865 & 0.00303038 & 0 \\
\hline 12 & -0.544259 & 0.00215509 & 0.000554959 \\
\hline 13 & 100 & 0.00680149 & 0 \\
\hline
\end{tabular}

TABLE 4 The aspherical coefficient of the ultra-wide angle lens design.

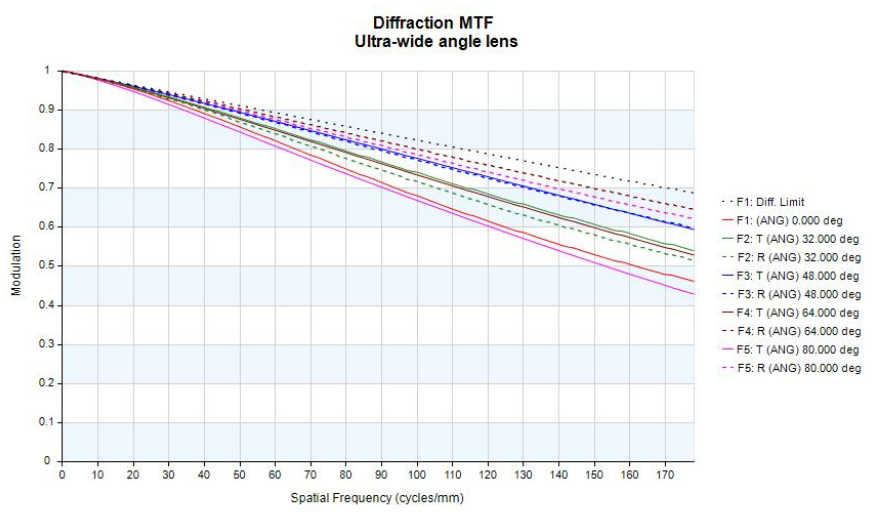

FIG. 6 MTF and spatial frequency at infinity object distance.

between the short wavelength and long wavelength imaging heights, it will cause color distortion. If the lateral chromatic aberration is less than $1.4 \mu \mathrm{m}$ per pixel, then the color difference cannot be discriminated by a sensor. Figure 8 presents the lateral color and the half field angle, where the red line is the longitudinal chromatic aberration curve from $486.13 \mathrm{~nm}$ to $656.27 \mathrm{~nm}$, with the maximum value of $1.4 \mu \mathrm{m}$ and the green line is the longitudinal chromatic aberration curve from $486.13 \mathrm{~nm}$ to $587.56 \mathrm{~nm}$, with the maximum value of $0.34 \mu \mathrm{m}$. These are less than $1.4 \mu \mathrm{m}$ per pixel. The image F- $\theta$ distortion, lateral chromatic aberration and relative illumination are shown in Figures 7 to 9.

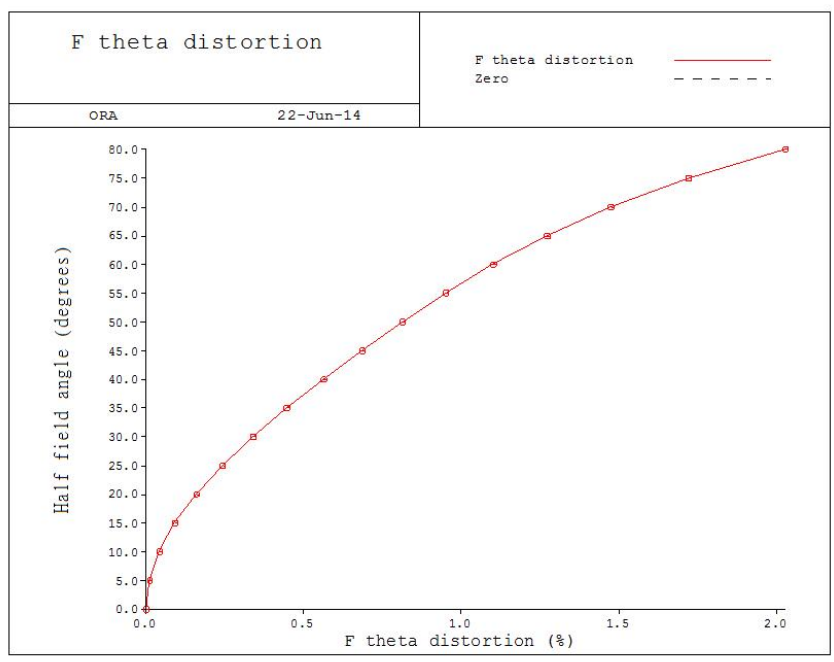

FIG. 7 F- $\theta$ distortion for the ultra-wide angle lens design.

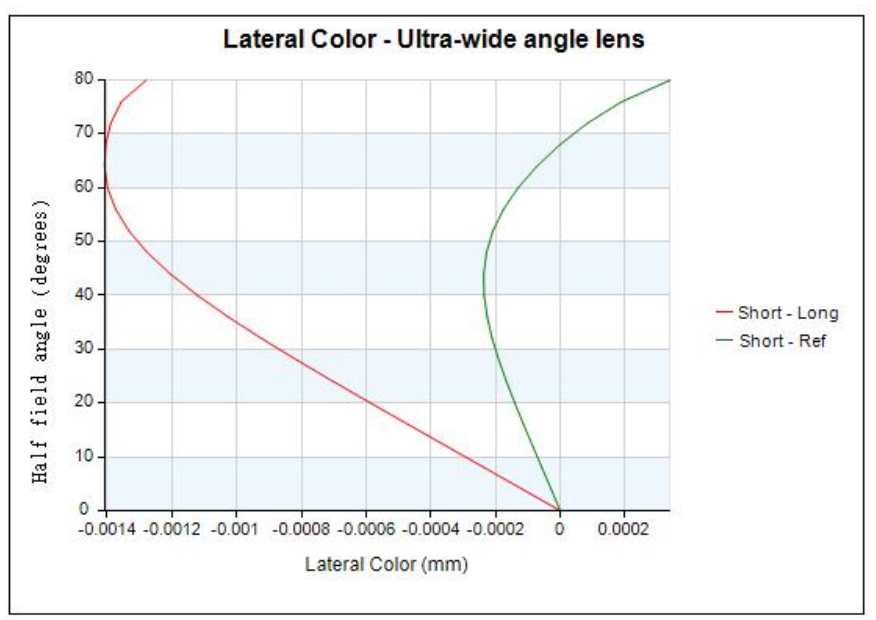

FIG. 8 Lateral color for the ultra-wide angle lens design.

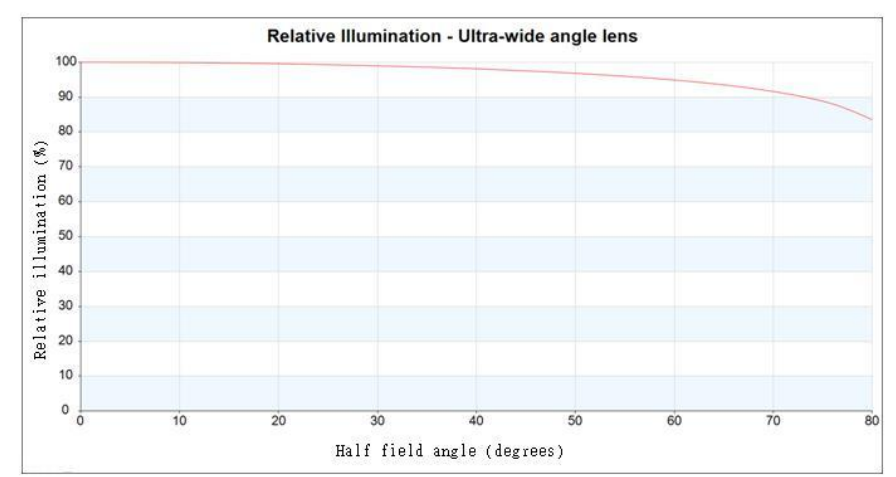

FIG. 9 Relative illumination for the ultra-wide angle lens design.

\section{CONCLUSION}

There are five spherical glass lenses and two aspheric plastic lenses and two flat glass plates in the optical system. The design result for the ultra-wide angle lens is a half field angle of $80^{\circ}, \mathrm{F} / 2.4$, a focal length $1.1 \mathrm{~mm}$, and a total length of $22.37 \mathrm{~mm}$. In the optimization process, we increase the $\left(N A_{Y} \times N A_{X} \times \cos ^{4} \Theta\right) /\left(N A_{0}\right)^{2}$ value so that we can achieve greater than $83 \%$ relative illumination. The F-theta distortion is less than $2 \%$ and lateral chromatic aberration is less than $1.4 \mu \mathrm{m}$. 


\section{ACKNOWLEDGEMENTS}

This study was sponsored by the Ministry of Science and Technology of the Republic of China, Taiwan, under contract numbers MOST 103-2622-E-035-023-CC3, 104-2221-E-035 -058 -MY2 and 104-2221-E-008-096.

\section{References}

[1] R. W. Wood, Physical optics (Macmillan, New York, 1911).

[2] W. N. Bond, "A wide angle lens for cloud recording," Philosophical Magazine 44, 999-1001 (1922).

[3] C. Beck, "Apparatus to photograph the whole sky," J. Sci. Instrum. 2, 135-139 (1924).

[4] R. Hill, "A lens for whole sky photography," in Proceedings to the $3^{\text {rd }}$ Optical Convention, 878-883 (Imperial College of Science and Technology, South Kensington, London, 1926).

[5] J. Y. Zheng, and S. Li, "Employing a fish-eye for scene tunnel scanning," in Proceedings to the $7^{\text {th }}$ Asian Conference on Computer Vision, 509-518 (AFCV, Hyderabad, India, 2006).

[6] W. Feng, B. Zhang, J. Röning, Z. Cao, and X. Zong, "An embedded omnidirectional vision navigator for automatic guided vehicles," Proc. SPIE 7878, 78780N (2011).

[7] Z. Huang, J. Bai, and X. Hou, "A multi-spectrum fish-eye lens for rice canopy detecting," Proc. SPIE 7849, 784912 (2010).

[8] W. Li, and Y. F. Li, "Single-camera panoramic stereo imaging system with a fisheye lens and a convex mirror," Opt. Express 19, 5855-5867 (2011).

[9] M. Laikin, "Wide angle lens systems," Proc. SPIE 0237, 530-533 (1980).

[10] W. Wöltche, "The influence of back focus determination on wideangle lens design," Proc. SPIE 0399, 210-216 (1983).

[11] C. L. Lin, "Wide angle lens module and vehicle vision system," U. S. Patent US7944626 (2011).

[12] A. Ning, "Compact fisheye objective lens," U. S. Patent US7023628 (2006).

[13] M. Kawada, “Fisheye lens unit,” U. S. Patent US7283312 (2007).

[14] G. I. Kweon, Y. H. Choi, and M. Laikin, "Fisheye lens for image processing applications," J. Opt. Soc. Korea 12, 79-87 (2008).

[15] Synopsys, Code V Electronic Document Library, Version 10.5, Lens System Setup for Reference Manuals, Chap. 1, p 35. (2012).

[16] E. T. Burle, Electro-optics handbook (second edition, McGraw-Hill, New York, 2000).

[17] M. P. Rimmer, "Relative illumination calculations," Proc. SPIE 655 , 99-104 (1986).

[18] R. Kingslake, Optical system design (Academic Press, New York, 1983). 